\title{
BMJ Open Cost-effectiveness of interventions to control cardiovascular diseases and type 2 diabetes mellitus in South Asia: protocol for a systematic review
}

To cite: Singh K, Chandra Sekaran AM, Bhaumik S, et al. Cost-effectiveness of interventions to control cardiovascular diseases and type 2 diabetes mellitus in South Asia: protocol for a systematic review. BMJ Open 2015;5:e007205. doi:10.1136/bmjopen-2014007205

- Prepublication history for this paper is available online. To view these files please visit the journal online (http://dx.doi.org/10.1136/ bmjopen-2014-007205)

Received 17 November 2014 Revised 17 February 2015 Accepted 19 February 2015

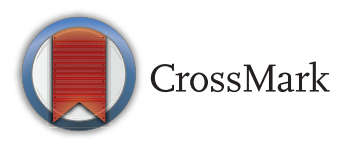

For numbered affiliations see end of article.

Correspondence to

Kavita Singh;

kavita@ccdcindia.org

Kavita Singh, ${ }^{1}$ Ambalam M Chandra Sekaran, ${ }^{2}$ Soumyadeep Bhaumik, ${ }^{3}$ Malini Aisola, ${ }^{4}$ Kaushik Chattopadhyay, ${ }^{5}$ Anuji U Gamage, ${ }^{6}$ Padmal de Silva, ${ }^{7}$ Sakthivel Selvaraj, ${ }^{4}$ Ambuj Roy, ${ }^{1}$ Dorairaj Prabhakaran, ${ }^{2,4}$ Nikhil Tandon ${ }^{1}$

\section{ABSTRACT}

Introduction: While a number of strategies are being implemented to control cardiovascular diseases (CVDs) and type 2 diabetes mellitus (T2DM), the costeffectiveness of these in the South Asian context has not been systematically evaluated. We aim to systematically review the economic (cost-effectiveness) evidence available on the individual-, group- and population-level interventions for control of CVD and T2DM in South Asia.

Methods and analysis: This review will consider all relevant economic evaluations, either conducted alongside randomised controlled trials or based on decision modelling estimates. These studies must include participants at risk of developing CVD/T2DM or with established disease in one or more of the South Asian countries (India, Bangladesh, Pakistan, Sri Lanka, Nepal, Maldives, Bhutan and Afghanistan). We will identify relevant papers by systematically searching all major databases and registries. Selected articles will be screened by two independent researchers.

Methodological quality of the studies will be assessed using a modified Drummond and a Phillips checklist. Cochrane guidelines will be followed for bias assessment in the effectiveness studies.

Results: Results will be presented in line with the PRISMA (Preferred Reporting Items for Systematic review and Meta-analysis) checklist, and overall quality of evidence will be presented as per the GRADE (Grades of Recommendation, Assessment, Development and Evaluation) approach.

Ethics and dissemination: The study has received ethics approval from the All India Institute of Medical Sciences, New Delhi, India. The results of this review will provide policy-relevant recommendations for the uptake of cost-effectiveness evidence in prioritising decisions on essential chronic disease care packages for South Asia.

Study registration number: PROSPERO CRD42013006479.

\section{INTRODUCTION}

Cardiovascular disease (CVD) and type 2 diabetes mellitus (T2DM) together contribute to the largest burden of morbidity (14\% of disability-adjusted life years) and mortality (over $30 \%$ of all deaths) in South Asia. ${ }^{1}{ }^{2}$ T2DM doubles the risk of developing CVD, and approximately half of patients with T2DM are known to be hypertensive. ${ }^{34}$ By 2030 , it is projected that there will be 120.9 million people with diabetes in South Asia (90-95\% of these will have T2DM), more than double the number affected in North America or Europe. ${ }^{2}$

South Asians experience higher case fatality rates and rates of premature death due to CVD (deaths occurring at least 10-15 years younger) than the rest of the Western world. ${ }^{56}$ A report in 2010 suggested that the total annual income loss to households affected by CVDs in India was $144-158$ billion INR. ${ }^{7}$ The WHO estimates that India will lose US\$237 billion due to heart disease, stroke and diabetes, which will slow the growth in India's GDP (gross domestic product) by $1 \%$ over the next 10 years, ${ }^{8}$ thereby contributing to poverty. The mortality and morbidity due to CVD/ T2DM thus impose a huge economic burden on individuals, families and society, the health system, and the economy as a whole. ${ }^{9} 10$

CVD and T2DM share various common risk factors (unhealthy diet, physical inactivity, tobacco use, high blood pressure, dyslipidaemia and stress), and hence there is considerable overlap in strategies used to control these diseases. ${ }^{4}$

While the efficacy and safety of various interventions have been tested by several randomised controlled clinical trials and subsequent systematic reviews and meta-analyses, little is known about the cost-effectiveness of these interventions from the perspective of either the patient or the healthcare system.

Rationale: why is a systematic

review needed?

Scarcity of resources in South Asia (Afghanistan, Bhutan, Bangladesh, India, 
Maldives, Nepal, Pakistan and Sri Lanka) has meant that policy-makers have to make active decisions about funding of interventions prioritised for inclusion in essential care packages to maximise benefit from the limited resources. ${ }^{11}$ Promoting effective care without considering the costs of care and the value of the health gain leads to inefficient use of public and private funds allocated for healthcare, indirectly resulting in harm to individuals and society rather than the intended good. ${ }^{12}$ Therefore, it is prudent to interpret treatment guidelines based on the safety and efficacy measures combined with the cost-effectiveness to help make decisions more patient-oriented. The recent emerging trends of prevention initiatives and national programmes to control CVD and T2DM in the South Asian region necessitate a renewed focus on economic aspects of interventions. The current review will fill this gap by systematically evaluating the cost-effectiveness of interventions in South Asia. Results from this review will enable policy-makers, clinicians and patient's advocacy groups in the resource-constrained South Asian setting to make better 'evidence-informed decisions'.

\section{Aim}

To systematically review the economic (costeffectiveness) evidence available on the individual-, group- and population-level interventions used for control of GVD and T2DM in South Asia.

\section{Specific objectives}

1. To summarise the cost-effectiveness evidence for interventions to control CVD and T2DM in South Asia.

2. To describe the quality of the economic evaluation studies considering the key methodological issues (time horizon, analytical viewpoint, outcome measures).

3. To summarise the types of incremental resource inputs, consequences and costs of implementing the intervention, versus comparators.

\section{METHODS AND ANALYSIS}

\section{Criteria for inclusion of studies in this review}

Type of studies

We will include full economic evaluation studies (ie, cost-effectiveness analyses, cost-utility analyses, costbenefit analyses) based on either randomised controlled trials or decision modelling-based cost-effectiveness studies. This approach is more inclusive and we recognise that different types of economic evaluations may involve heterogeneity; therefore, we will classify and analyse them separately in order to retain comparability among a particular type of economic evaluation.

\section{Type of participants}

Studies that include individuals at risk (as defined by the study authors) of developing T2DM or CVD and/or with established CVD or T2DM receiving treatment will be included. Also, the economic evaluations must include participants in one or more of the South Asian countries: India, Bangladesh, Pakistan, Sri Lanka, Nepal, Maldives, Bhutan and Afghanistan.

\section{Types of interventions}

We reviewed the current national health policy documents of South Asian countries to understand and compile a list of strategies or interventions currently being recommended to control CVD and T2DM in the region. Table 1 summarises the results of this exercise. In addition, clinical guidelines for T2DM and CVD management will be screened to identify the individual-, group- and population-level interventions proposed in table 2 .

It will be of great value to summarise the evidence on cost-effectiveness as the first step in promoting the costeffective strategies for prevention and control of CVD and T2DM. This will help make healthcare decisions more accountable.

\section{Types of outcome measures}

We will report outcome measures as summarised in table 3 , if available in the published articles. If relevant information is lacking, attempts will be made to obtain maximum data on these outcome measures by contacting primary authors.

\section{Exclusion criteria}

1. Non-economic evaluation studies.

2. Reviews, letters, abstracts, methodological and general commentary or perspectives.

3. Economic evaluations reported for non-South Asian countries.

4. Studies without English language titles and abstracts.

\section{Locating studies and search strategy}

The search for relevant studies will be carried out by two independent reviewers. We will search the following electronic databases: (1) The Cochrane Library; (2) The Database of Abstracts of Reviews of Effectiveness (DARE) - contained in the Cochrane library search; (3) Medline (1966+); (4) EMBASE (1980+), (5) National Health Service Economic Evaluation Database (NHS EED); (6) Health EED (HEED); (7) Centre for Reviews and Dissemination (CRD) electronic database; (8) The CEA Registry; (9) Econlit; (10) CINAHL; (11) Biomed central; (12) PsychInfo; (13) Science Direct; (14) Web of Science.

Relevant publications of the DCP2 (Disease Control Priority 2) project and WHO-CHOICE programme will be completely hand-searched for relevant articles. As the use of technical terms for indexing international literature in databases is often inconsistent or errant, we will define a search strategy with high sensitivity. The search strategy will consist of freetext and $\mathrm{MeSH}$ terms related to economic evaluation and CVD or T2DM. Further, the 
Table 1 Strategies or interventions recommended for cardiovascular disease (CVD) and type 2 diabetes mellitus (T2DM) in the national policy documents

\section{No Countries Health policy documents (source)}

1. Afghanistan National Strategy for Prevention and Control of Non-communicable diseases (NCDs), 2013-2018; ${ }^{13}$ endorsed by Ministry of Public Health Afghanistan, General Directorate of Preventive Medicine NCD Prevention and Control Department

2. Bhutan

National Policy and Strategic Frame-Work on Prevention and Control of $\mathrm{NCDs}^{14}$ (Endorsed by the Royal Government of Bhutan 2009)

3. Bangladesh Strategic Plan for Surveillance and Prevention of NCDs in Bangladesh, 2007-2010 ${ }^{15}$ (endorsed by Ministry of Health and Family Welfare)

4. India

National Programme for Prevention and Control of Cancer, Diabetes, CVDs and Stroke (NPCDCS), $2010^{16}$

5. Maldives

National Strategic Plan for Prevention and Control of NCDs 2008-2010 ${ }^{17}$

6. Nepal

Integrated NCD Prevention and Control Policy of Nepal, 2007-08 ${ }^{18}$

7. Pakistan

\section{Recommended interventions/strategies}

(1) Introduce interventions targeted to reduce tobacco use, unhealthy diet, physical inactivity, and harmful use of alcohol; (2) strengthen health system and integrate NCD programme in PHC; (3) develop and implement effective NCD advocacy plan; (4) health promotion, media campaigns, workplace-based programmes, and promote

population-based interventions; (5) build capacity of healthcare workers; (6) establish national diabetes registry and surveillance of NCD risk factors (STEPS survey); (7) establish multisectoral partnerships

(1) Introduce alcohol and tobacco taxes; (2) introduce interventions to improve physical activity in schools and community; (3) promote healthy lifestyle initiatives; (4) strengthen health services to provide timely treatment and continuum of care

(1) Health facility strengthening; (2) capacity building; (3) availability of essential drugs; (4) screening of high-risk individuals; (5) development of surveillance system

(1) Prevention through behaviour change: mass media, community education, and interpersonal communication to be used for increased intake of healthy food, increased physical activity, avoidance of tobacco and alcohol, and stress management; (2) opportunistic screening of those individuals at high risk of developing T2DM and CVD; (3) range of treatment services: health promotion, psychosocial counselling, management (out- and in-patient), day care services, home-based care and palliative care, and referral to specialised services

(1) Encourage healthy lifestyles in school and community setting-for example, tobacco-free islands; (2) awareness campaigns and health education session; (3) develop and disseminate treatment guidelines for major NCDs; (4) conduct screening in high-risk groups; (5) integrate and strengthen NCD management in PHC; (6) build capacity for care providers

(1) Reduce tobacco use and alcohol consumption: 'sin tax'; (2) establish NCD surveillance system; (3) build capacity for healthcare workers; (4) prioritise low-cost, cost-effective socio-culturally acceptable measures in planning and implementation of NCD prevention and control

CVD and T2DM action plan: (1) integrate surveillance of CVD risk factors with population-based NCD surveillance system; (2) promote physical activity and healthy diet; (3) agricultural and fiscal policies to increase access to healthy food; (4) population-level screening of risk factors; (5) ensure availability of aspirin, $\beta$-blockers, thiazides, ACE inhibitors, statins and penicillin at all levels of healthcare; (6) ensure availability of antidiabetic agents (insulin, sulfonylureas, metformin) at all levels of healthcare; (7) build capacity of health systems in support of CVD prevention and control 


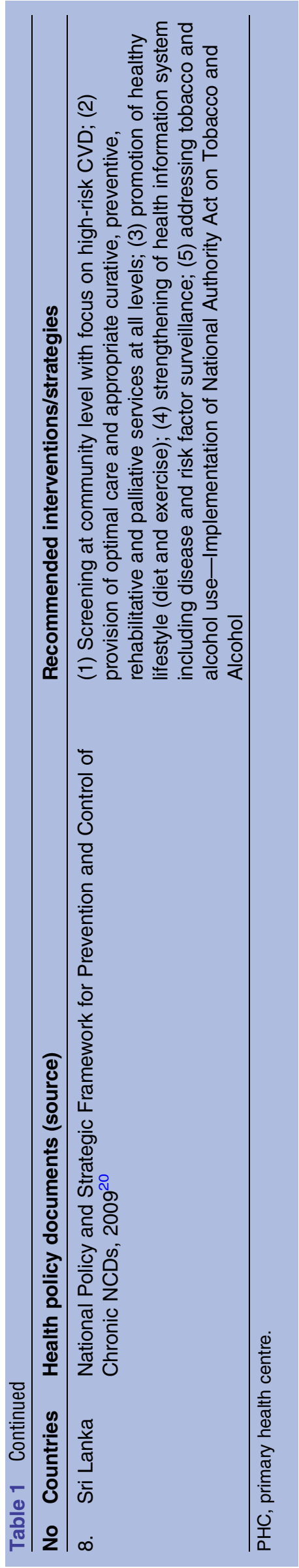

resulting hits will be filtered for the occurrence of the term 'South Asian countries'. The search will include all years up to 2014. Further the reference lists of identified relevant studies and reviews will be hand-searched.

Keywords that will be used for building the search strategy include:

- CVD OR T2DM: (heart disease, ischaemic heart disease, myocardial infarction, congestive heart failure, hypertension, cardiac disease, revascularisation, cardiac rehabilitation, diabetes, DM, T2DM, impaired fasting glucose, impaired glucose tolerance, pre-diabetes) $A N D$

- Cost-effectiveness: (Costs OR (Cost analysis or costanalyses) OR (Cost-effective) OR (Cost-benefit) OR (Cost-utility) OR (Cost-minimisation) OR (Health expenditure) OR (Cost estimate)) AND

- South Asian countries: (Afghanistan OR Bangladesh OR Bhutan OR India OR Maldives OR Nepal OR Pakistan OR Sri Lanka)

\section{Data collection}

Screening of studies for eligibility

We will import search results into Zotero and remove duplicates as a first step. Screening will then be conducted in two phases. First, titles and abstracts will be screened by one researcher to identify publications that definitely do not meet the inclusion criteria. In doubtful cases, the publication will be included. Second, the full text of the selected publications will be screened by two independent researchers to match the eligibility criteria. Any disagreements will be resolved by consensus discussion within the study team. The inter-rater reliability for inclusion of economic studies will be calculated and reported using $\kappa$ statistics. Tables of excluded studies will be prepared, detailing when exclusion occurred and the reasons for exclusion. A predefined data extraction form will be used for this task. The form will be developed and piloted on five economic studies to refine it before being used on all studies. Records of all studies will be kept as per the PRISMA (Preferred Reporting Items for Systematic review and Meta-analysis) checklist. ${ }^{21}$

\section{Collecting data}

We will collect information on characteristics of included studies and their results. To describe the characteristics of included studies, we will extract: year of study; details of interventions and comparators; study design and source(s) of resource use, unit costs and (if applicable) effectiveness data; decision-making jurisdiction, geographical and organisational setting; analytical viewpoint; and time horizon for both costs and effects. Where information is missing, we will contact study authors to request additional details.

For outcome measures, estimates of specific items of resource use associated with interventions and comparators, along with their unit costs, will be extracted in natural units (eg, length of hospital stay in days, duration of operation in minutes, number of outpatient attendances, etc). We will also collect information on the price year and 
Table 2 Interventions to be evaluated in this review (not exhaustive)

\begin{tabular}{|c|c|c|c|c|}
\hline \multicolumn{4}{|c|}{ Pharmacological interventions } & \multirow{2}{*}{$\begin{array}{l}\text { Surgical and percutaneous } \\
\text { interventions } \\
\text { Procedures }\end{array}$} \\
\hline $\begin{array}{l}\text { Blood pressure-lowering } \\
\text { drugs }\end{array}$ & Antiplatelet inhibitors & Lipid-lowering drugs & $\begin{array}{l}\text { Oral hypoglycaemic } \\
\text { agents }\end{array}$ & \\
\hline \multicolumn{5}{|c|}{ Individual-based interventions } \\
\hline $\begin{array}{l}\text { Angiotensin receptor } \\
\text { blocker }\end{array}$ & Clopidogrel & Simvastatin & Sulfonylureas & Angioplasty \\
\hline Calcium channel blockers & & Fibrates & Insulin & Cardiac rehabilitation \\
\hline$\beta$-Blockers & & & & $\begin{array}{l}\text { Cardiac resynchronisation therapy } \\
\text { (CRT) }\end{array}$ \\
\hline $\begin{array}{l}\text { Polypill (fixed dose } \\
\text { combination cardiovascular } \\
\text { polypill) }\end{array}$ & & & & $\begin{array}{l}\text { Procedures to treat complications of } \\
\text { diabetes: amputation, laser } \\
\text { photocoagulation therapy etc. }\end{array}$ \\
\hline \multicolumn{5}{|c|}{ Group/population-based interventions } \\
\hline Health education & $\begin{array}{l}\text { Health financingMultisectoral } \\
\text { approach (eg, agricultural policy) } \\
\text { Sin tax }\end{array}$ & $\begin{array}{l}\text { High-risk screeningOpportunistic } \\
\text { screeningGeneral/whole population } \\
\text { screening }\end{array}$ & $\begin{array}{l}\text { Peer-support } \\
\text { interventions }\end{array}$ & $\begin{array}{l}\text { Lifestyle behavioural counselling } \\
\text { targeted at individual or } \\
\text { population-based strategy: } \\
\text { - Physical activity } \\
\text { - Diet } \\
\text { - Tobacco cessation } \\
\text { - Alcohol consumption }\end{array}$ \\
\hline
\end{tabular}


Table 3 Outcome measures to be reported in this review

\begin{tabular}{|c|c|c|}
\hline Resource use & Costs & Cost-effectiveness \\
\hline $\begin{array}{l}\text { No of outpatient } \\
\text { attendances }\end{array}$ & Direct medical costs & Incremental cost-effectiveness ratios \\
\hline $\begin{array}{l}\text { No of inpatient } \\
\text { hospitalisations }\end{array}$ & Indirect medical costs & Cost per life years gained \\
\hline $\begin{array}{l}\text { Length of hospital stay } \\
\text { in days }\end{array}$ & $\begin{array}{l}\text { Out-of-pocket expenses paid by } \\
\text { the participants/patients }\end{array}$ & Cost per QALYs (quality-adjusted life years) gained \\
\hline $\begin{array}{l}\text { Other direct medical } \\
\text { resource use }\end{array}$ & $\begin{array}{l}\text { Costs of materials and } \\
\text { intervention delivery (training } \\
\text { cost) }\end{array}$ & Cost per DALYs (disability-adjusted life years) averted \\
\hline $\begin{array}{l}\text { Other indirect medical } \\
\text { resource use }\end{array}$ & & $\begin{array}{l}\text { Cost per unit reduction in risk factors (such as blood pressure, } \\
\text { blood sugar, HbA1c, low-density lipoprotein cholesterol) }\end{array}$ \\
\hline
\end{tabular}

currency used to calculate incremental cost estimates. Both a point estimate and a measure of uncertainty (eg, SE or CI) will also be extracted for measures of incremental resource use, costs and cost-effectiveness, if reported. In addition, details of any sensitivity analyses undertaken will be collected.

\section{Assessment of study quality}

We will assess whether the published studies have described economic analyses methods, assumptions, models and possible biases in a way that is transparent, so that the strength of economic studies can be determined. Since the reliability of an economic evaluation is predicated on its use of reliable effectiveness data, part of the critical appraisal will involve considering sources of potential bias that apply to the randomised controlled trial.

In this review, the critical appraisal will therefore consist of the following three elements:

1. Assessment of the risk of bias in results of the effectiveness studies (randomised controlled trial), using Cochrane guidelines.

2. Assessment of the methodological quality of the economic evaluations, using a modified Drummond checklist $^{22}$ and Evers checklist. ${ }^{23}$

3. Assessment of the methodological quality of decision modelling studies, using Phillips checklist 2004. ${ }^{24}$

In general, factors that will be assessed for methodological quality are those related to applicability of findings, validity of individual studies, and certain design characteristics that affect interpretation of results, such as double blinding and adherence. ${ }^{25}{ }^{26}$ Further, four sources of bias will be checked in primary effectiveness studies: selection bias, performance bias, attrition bias and detection bias. Two reviewers will independently assess the methodological quality of selected studies. It is plausible that use of different data sources for measures of resource use, cost and/or cost-effectiveness will impact on results; therefore, sensitivity analysis will be performed to assess how the outcomes measures are influenced by adding some of the excluded economic studies that did not meet the minimum quality requirements of a good-quality economic evaluation.
Analysing, interpreting and reporting results

Presenting results in tables and narrative summary

We will use appropriate analytical methods for summarising the results of this review. If applicable, a meta-analysis of resource use or cost data, or development of an economic model, may be considered. In addition to reporting the characteristics of included studies, a summary table of various checklists completed to inform assessments of the methodological quality of economic evaluations will be presented. Also, we will report a commentary on the main characteristics and results of included studies (measures of incremental resource use, cost and cost-effectiveness).

Costs will be presented in real currency (as of the year of study or adjusted to current year) as this will be relevant for the readers in the countries under study. In addition, in order to facilitate comparison of cost estimates collected from different studies, an international exchange rate based on purchasing power parities (PPPs) will be used to convert cost estimates into a target currency - that is, international dollars - and GDP deflators will be used to convert cost estimates into a fixed price year.

\section{Addressing reporting and publication biases}

Publication bias will be detected by a funnel plot, if there are more than 10 studies in a particular intervention category. If asymmetry is seen, this will be discussed to consider reasons other than publication bias-for example, selection bias, reporting bias, data irregularities, true heterogeneity and artefacts. Subgroup analysis will be performed to determine whether benefit varies across intervention types or countries.

Since English is the dominant language for health research in South Asia, exclusion of non-English language studies will introduce small bias.

\section{Heterogeneity and sensitivity analyses}

We will test heterogeneity of intervention effects among trials using the standard $\chi^{2}$ statistic ( $\mathrm{p}$ value) or the $\mathrm{I}^{2}$ statistic. We will consider a $p$ value of $>0.10$ to be statistically significant heterogeneity. Interpretation of $\mathrm{I}^{2}$ for 
heterogeneity is as follows: $0-40 \%$, may not be important; $30-60 \%$, represents moderate heterogeneity; $50-$ $90 \%$, represents substantial heterogeneity; $75-100 \%$, represents considerable heterogeneity. ${ }^{27}$ We will explore the possible cause(s) of heterogeneity by conducting various sensitivity analyses.

\section{Summary of findings}

Results of this review will be reported in line with the PRISMA 2009 checklist. $^{28}$ The overall quality of evidence on outcomes will be presented using the GRADE (Grades of Recommendation, Assessment, Development and Evaluation) approach, ${ }^{29}$ which involves consideration of within-study risk of bias (methodological quality), directness of evidence, heterogeneity, precision of effect estimates and risk of publication bias. We will rate overall quality of evidence at four levels: high, moderate, low and very low.

\section{Ethics and dissemination}

The systematic review protocol has been approved by the institutional ethics committee of the All India Institute of Medical Sciences, New Delhi, India. As such, there are no ethical issues involved in this study, as it is only a review of published economic evaluations and no patient data will be collected.

Findings from this review will be submitted for publication in peer-reviewed journals. They will be shared with decision-makers and health professionals as brief policy notes. The study investigators will also disseminate findings through professional conferences, targeting primary and secondary care physicians, health economists, and public health policy-makers more widely. While there has been an increasing interest in South Asian countries to scale-up the most cost-effective individual-, group- and population-level interventions for CVD and T2DM management, major research gaps will be identified through this review. The results of this study will provide policyrelevant recommendations for the uptake of costeffectiveness evidence in prioritising decisions on essential chronic disease care packages in South Asia.

\section{Author affiliations \\ ${ }^{1}$ All India Institute of Medical Sciences, New Delhi, India \\ ${ }^{2}$ Centre for Chronic Disease Control, Gurgaon, Haryana, India \\ ${ }^{3}$ BioMedical Genomics Centre, Kolkata, India \\ ${ }^{4}$ Public Health Foundation of India, Institute for Studies in Industrial Development Campus, New Delhi, India \\ ${ }^{5}$ London School of Hygiene and Tropical Medicine, London, UK \\ ${ }^{6}$ Nutrition Division, Ministry of Health, Colombo, Sri Lanka \\ ${ }^{7}$ Department of Research, WHO Collaborating Centre on Public Health Workforce Development, National Institute of Health Sciences, Kalutara, Sri Lanka}

Acknowledgements Kavita Singh, Padmal De Silva and Anuji Gamage are supported by the Fogarty International Centre, National Institutes of Health, under Award Number: D43TW008332 (ASCEND Research Network). The contents of this publication is solely the responsibility of the author/s and does not necessarily represent the official views of the National Institutes of Health or the ASCEND Research Network.
Contributors KS and SB drafted the manuscript. All authors contributed to the conception and design of the protocol and critical review for intellectual content, and approved the final version of the manuscript submitted for publication.

Competing interests None.

Ethics approval All India Institute of Medical Sciences, New Delhi approved this study as part of the doctoral thesis work.

Provenance and peer review Not commissioned; externally peer reviewed.

Open Access This is an Open Access article distributed in accordance with the Creative Commons Attribution Non Commercial (CC BY-NC 4.0) license, which permits others to distribute, remix, adapt, build upon this work noncommercially, and license their derivative works on different terms, provided the original work is properly cited and the use is non-commercial. See: http:// creativecommons.org/licenses/by-nc/4.0/

\section{REFERENCES}

1. Ohira T, Iso H. Cardiovascular disease epidemiology in Asia: an overview. Circ J 2013;77:1646-52.

2. Mathers $C D$, Loncar $D$. Projections of global mortality and burden of disease from 2002 to 2030. PLoS Med 2006;3:e442.

3. Nag T, Ghosh A. Cardiovascular disease risk factors in Asian Indian population: a systematic review. J Cardiovasc Dis Res 2013;4:222-8.

4. Ramachandran A, Snehalatha C, Latha E, et al. Clustering of cardiovascular risk factors in urban Asian Indians. Diabetes Care 1998;21:967-71.

5. Ghaffar A, Reddy KS, Singhi M. Burden of non-communicable diseases in South Asia. BMJ 2004;328:807-10.

6. Hughes LO, Raval U, Raftery EB. First myocardial infarctions in Asian and white men. BMJ 1989:298:1345-50.

7. Ajay M, Karan A, Engelgau M. The Economic Implications of Non-Communicable Disease for India, Health, Nutrition and Population (HNP) Discussion Paper, the World Bank. 2010.

8. WHO. Preventing chronic diseases: a vital investment: WHO global report. Geneva: World Health Organization, 2005.

9. Economic Intelligence Unit. The silent epidemic: an economic study of diabetes in developed and developing countries. New York, London, Hong Kong: The Economist, 2007.

10. Xu K, Evans DB, Kawabata K, et al. Household catastrophic health expenditure: a multicountry analysis. Lancet 2003;362:111-17.

11. Lavis J, Davies H, Oxman A, et al. Towards systematic reviews that inform health care management and policy-making. $J$ Health Serv Res Policy 2005;10(Suppl 1):35-48.

12. Williams A. Health economics: the cheerful face of the dismal science? In: Williams A, ed. Health and economics. London, UK: Macmillan, 1987.

13. National Strategy for Prevention and Control of Non-communicable diseases (NCDs), 2013-2018. http://moph.gov.af/Content/Media/ Documents/NCDStrategy_Draft_Final812201 3104122676 553325325.docx (accessed 18 Jan 2014).

14. Bhutan National Policy and Strategic Frame-Work on Prevention and Control of Non Communicable Diseases. http://www.health.gov.bt/ downloads/NationalNCD.pdf (accessed 14 Apr 2014).

15. Strategic Plan for Surveillance and Prevention of Non-Communicable Diseases in Bangladesh, 2007-2010. http:// www.nationalplanningcycles.org/../Bangladesh/bangladesh_stratigic plan_ncd_2007-2010_pdf (accessed 14 Apr 2014).

16. National Programme for Prevention and Control of Cancer, Diabetes, Cardiovascular Diseases \& Stroke (NPCDCS), 2010 - India. health. bih.nic.in /Docs/Guidelines/Guidelines-NPCDCS.pdf (accessed 14 Apr 2014)

17. National strategic plan for prevention and control of Non-Communicable Diseases 2008-2010. http://siteresources. worldbank.org/SOUTHASIAEXT/Resources/2235461296680097256/7707437-1296680114157/ NCD_MV_Policy_Feb_2011.pdf (accessed 24 Jan 2014).

18. Integrated Non-Communicable Diseases (NCDs) Prevention and Control Policy of Nepal, 2007-08. http://mohp.gov.np/english/files/ new_publications/

9-4-Integrated-Non-comunicable-Disease-Prevention-and-contro.pdf (accessed 14 Apr 2014).

19. Nishtar S. The national action plan for the prevention and control of non-communicable diseases and health promotion in PakistanPrelude and finale. J Pak Med Assoc 2004;54:S1-8.

20. The National Policy \& Strategic Framework for Prevention and Control of Chronic Non-Communicable Diseases, Sri Lanka 2009. 
http://www.health.gov.Ik/en/NCD/temp/NCD\%20Policy\%20English. pdf (accessed 14 Apr 2014).

21. Pal S, Dutta S, Adhikary SS, et al. Hemi body irradiation: an economical way of palliation of pain in bone metastasis in advanced cancer. South Asian J Cancer 2014;3:28-32.

22. Drummond MF, Jefferson TO. Guidelines for authors and peer reviewers of economic submissions to the BMJ. The BMJ Economic Evaluation Working Party. BMJ 1996;313:275-83.

23. Evers $\mathrm{S}$, Goossens $\mathrm{M}$, de Vet $\mathrm{H}$, et al. Criteria list for assessment of methodological quality of economic evaluations: consensus on Health Economic Criteria. Int J Technol Assess Health Care 2005;21:240-5.

24. Kumar A, Sivakanesan R, Gunasekera S. Ischemia modified albumin: a potent marker in acute myocardial infarction in normolipidaemic. Pak J Med Sci 2008;24:364-7.
25. Neumann PJ, Greenberg D, Olchanski NV, et al. Growth and quality of the cost-utility literature, 1976-2001. Value Health 2005;8:3-9.

26. Rennie D, Luft HS. Pharmacoeconomic analyses: making them transparent, making them credible. JAMA 2000;283:2158-60.

27. Deeks JJ, Higgins JPT and Altman DG; on behalf of the Cochrane Statistical Methods Group. Analysing data and undertaking meta-analyses. hiv.cochrane.org/sites/hiv.cochrane.org/files/../Ch09_ Analysing.pdf (accessed 15 Mar 2014).

28. Haque U, Overgaard HJ, Clements AC, et al. Malaria burden and control in Bangladesh and prospects for elimination: an epidemiological and economic assessment. Lancet Glob Health 2014;2:e98-105.

29. Kwatra G, Kaur P, Toor G, et al. Cost of stroke from a tertiary center in northwest India. Neurol India 2013;61:627-32. 